\title{
SYMMETRIC OPERATORS WITH SINGULAR SPECTRAL FUNCTIONS
}

\author{
RICHARD C. GILBERT
}

In a previous article [3] it was shown that if $A$ is a closed symmetric operator with deficiency indices $(1,1)$ in the Hilbert space $H$ and if $\mu_{0}$ is a point of regular type for $A$, then there is a neighborhood of $\mu_{0}$ in which every minimal selfadjoint dilation $A^{+}$of $A$ has spectral multiplicity not more than 1 . This shows that in Theorem 14 of Coddington [2] and Theorem 5.2 of McKelvey [6] it is the hypothesis that every point is of regular type which determines the spectral multiplicity of the dilation; the hypothesis that the contraction $F(\lambda)$ is continuous down to the real axis and has norm less than 1 there serves to make the spectral function absolutely continuous. (See Remark 2.)

The procedure used in [3] depended on the fact that if $\mu_{0}$ is a point of regular type for $A$, then there exists a selfadjoint extension $A_{0}$ of $A$ for which $\mu_{0}$ is in the resolvent set. This means that for different points $\mu_{0}$ one might have to choose different operators $A_{0}$. In the present note this necessity is eliminated. It is shown that if $A$ has a selfadjoint extension $A_{0}$ with a pure point spectrum with no finite limit points, then for each minimal selfadjoint extension or dilation $A^{+}$of $A$ there is defined on the real axis a nondecreasing function $\rho(\sigma)$ (which depends solely on $A^{+}$and on the single selfadjoint extension $A_{0}$ ) such that $A^{+}$is unitarily equivalent to the multiplication operator in $L_{\rho}^{2}$. Kreln [5] has shown that if $A$ is simple, then there exists a selfadjoint extension $A_{0}$ of the type described above if and only if every point is of regular type for $A$. (See Remark 1 also.)

In the case that $A$ is a singular Sturm-Liouville operator, the results of the paper are extended to the case that there exists a selfadjoint extension $A_{0}$ with a singular spectral function.

For terminology used we refer the reader to [3] and to Achieser and Glasmann [1].

We note first of all the following lemma due to Straus [8].

Lemma 1. Suppose $\Phi(\lambda)$ is analytic in the upper halfplane with nonnegative imaginary part. Suppose $\Psi(\lambda)$ is analytic in some open set containing the real interval $[\alpha, \beta]$. Then, 1968.

Presented to the Society, March 4, 1968; received by the editors February 29. 


$$
\begin{aligned}
(2 \pi i)^{-1} & \lim _{\tau \rightarrow+0} \int_{\alpha}^{\beta}\{\Phi(\sigma+i \tau) \Psi(\sigma+i \tau)-[\Phi(\sigma+i \tau)]-\Psi(\sigma-i \tau)\} d \sigma \\
& =\int_{\alpha}^{\beta} \Psi(\sigma) d \rho(\sigma), \text { where } \rho(\sigma)=(1 / \pi) \lim _{\tau \rightarrow+0} \int_{0}^{\sigma} I \Phi(\sigma+i \tau) d \sigma .
\end{aligned}
$$

Here $I$ stands for the imaginary part and [ ]- stands for complex conjugate.

We note further the following lemma due to Kreln. (See Achieser and Glasmann [1, Appendix I, \$4].)

Lemma 2. Suppose that $A$ is a closed symmetric operator with deficiency indices $(1,1)$ in the Hilbert space $H$. Let $A_{0}$ be any selfadjoint extension of $A$. Let $g(\lambda)=g_{0}+\left(\lambda-\lambda_{0}\right) R_{0}(\lambda) g_{0}$, where $\lambda_{0}$ is a complex number with positive imaginary part; $g_{0}$ is an element of norm 1 in the deficiency subspace of $A$ corresponding to $\bar{\lambda}_{0} ; R_{0}(\lambda)$ is the resolvent of $A_{0}$. Let $Q_{1}(\lambda)=i \lambda_{0}+\left(\lambda-\lambda_{0}\right)\left(g_{0}, g(\bar{\lambda})\right)$. Then for $\Lambda \neq 0$ the generalized resolvent $R(\lambda)$ of $A$ corresponding to a selfadjoint extension or dilation $A^{+}$of $A$ has the form

$$
R(\lambda)=R_{0}(\lambda)-\left[\theta(\lambda)+Q_{1}(\lambda)\right]^{-1}(\cdot, g(\bar{\lambda})) g(\lambda),
$$

where $\theta(\lambda)$ is an analytic function for $\lambda \neq 0$ which has nonnegative imaginary part in the upper half-plane. $R(\lambda)$ corresponds to a selfadjoint extension in the space $H$ if and only if $\theta(\lambda)$ is identically equal to a constant $\theta$, where $-\infty<\theta \leqq+\infty$. The case $\theta=+\infty$ gives the resolvent $R_{0}(\lambda)$ corresponding to $A_{0}$.

Theorem. Let $A$ be a simple closed symmetric operator with deficiency indices $(1,1)$ in the Hilbert space $H$. Let $A_{0}$ be a selfadjoint extension of $A$ with a pure point spectrum having no finite limit points. Let $A^{+}$be a minimal selfadjoint extension of $A$ with corresponding generalized resolvent of $A$ represented as in Lemma 2 . Then, $A^{+}$is unitarily equivalent to the multiplication operator in $L_{\rho}^{2}$, where

$$
\begin{aligned}
& \rho(\sigma)=(1 / \pi) \lim _{\tau \rightarrow+0} \int_{0}^{\sigma} I \Phi(\mu+i \tau) d \mu, \\
& \Phi(\lambda)=\left[\theta(\lambda) Q_{1}(\lambda)-1\right]\left[\theta(\lambda)+Q_{1}(\lambda)\right]^{-1} .
\end{aligned}
$$

If $E^{+}(\lambda)$ is the spectral function of $A^{+}$, then for all elements $f, h \in H$,

(4) $\left(\left[E^{+}(\beta)-E^{+}(\alpha)\right] f, h\right)=\int_{[\alpha, \beta)}(f, g(\sigma))(g(\sigma), h)\left[Q_{1}^{2}(\sigma)+1\right]^{-1} d \rho(\sigma)$, where the integral is a Lebesgue-Stieltjes integral. 
Proof. For any $f, h$ in $H$, Krein's formula (1) for the generalized resolvent $R(\lambda)$ corresponding to $A^{+}$can be written in the form

$$
(R(\lambda) f, h)=F_{1}(\lambda)+F_{2}(\lambda)\left[\theta(\lambda) Q_{1}(\lambda)-1\right]\left[\theta(\lambda)+Q_{1}(\lambda)\right]^{-1},
$$

where

$$
\begin{aligned}
F_{1}(\lambda)=\left\{( R _ { 0 } ( \lambda ) f , h ) \left[Q_{1}^{2}(\lambda)\right.\right. & +1] \\
& \left.-Q_{1}(\lambda)(f, g(\bar{\lambda}))(g(\lambda), h)\right\}\left[Q_{1}^{2}(\lambda)+1\right]^{-1}
\end{aligned}
$$

and

$$
F_{2}(\lambda)=(f, g(\bar{\lambda}))(g(\lambda), h)\left[Q_{1}^{2}(\lambda)+1\right]^{-1} .
$$

We claim that $F_{1}(\lambda)$ and $F_{2}(\lambda)$ are entire functions of $\lambda$.

In order to establish this claim, we observe that the following equations are true:

$$
\begin{aligned}
\left(R_{0}(\lambda) f, h\right) & =\int_{-\infty}^{\infty}(\mu-\lambda)^{-1} d\left(E_{0}(\mu) f, h\right) ; \\
(f, g(\bar{\lambda}))= & \left(f, g_{0}\right)+\left(\lambda-\bar{\lambda}_{0}\right) \int_{-\infty}^{\infty}(\mu-\lambda)^{-1} d\left(E_{0}(\mu) f, g_{0}\right) ; \\
(g(\lambda), h)= & \left(g_{0}, h\right)+\left(\lambda-\lambda_{0}\right) \int_{-\infty}^{\infty}(\mu-\lambda)^{-1} d\left(E_{0}(\mu) g_{0}, h\right) ; \\
Q_{1}(\lambda)= & i \lambda_{0}+\left(\lambda-\lambda_{0}\right) \\
& +\left(\lambda-\lambda_{0}\right)\left(\lambda-\bar{\lambda}_{0}\right) \int_{-\infty}^{\infty}(\mu-\lambda)^{-1} d\left(E_{0}(\mu) g_{0}, g_{0}\right),
\end{aligned}
$$

where $E_{0}(\mu)$ is the spectral function of $A_{0}$.

If $\mu_{0}$ is a point in the resolvent set of $A_{0}$, then each of the functions $\left(R_{0}(\lambda) f, h\right),(f, g(\bar{\lambda})),(g(\lambda), h), Q_{1}(\lambda)$ is an analytic function of $\lambda$ in a neighborhood of $\mu_{0}$. Further, $Q_{1}(\lambda)$ is real for $\lambda$ real, so that $Q_{1}^{2}(\lambda)+1$ is not zero in this neighborhood for $\lambda$ sufficiently close to the real axis. It follows that $F_{1}(\lambda)$ and $F_{2}(\lambda)$ are analytic in a neighborhood of $\mu_{0}$.

Suppose $\mu_{0}$ is an eigenvalue of $A_{0}$. Since $A$ is simple, $g_{0}$ is a generating element for $A_{0}$. (See Achieser and Glasmann [1, $\$ 81$, Theorem 2].) Hence we may write equations (8), (9), (10), (11) in the form

$$
\begin{aligned}
\left(R_{0}(\lambda) f, h\right)= & G_{1}(\lambda) \\
& +\left(\mu_{0}-\lambda\right)^{-1}\left(f, E_{0}\left[\mu_{0}\right] g_{0}\right)\left(E_{0}\left[\mu_{0}\right] g_{0}, h\right)\left(E_{0}\left[\mu_{0}\right] g_{0}, g_{0}\right)^{-1}, \\
(f, g(\bar{\lambda}))= & G_{2}(\lambda)+\left(\lambda-\bar{\lambda}_{0}\right)\left(\mu_{0}-\lambda\right)^{-1}\left(f, E_{0}\left[\mu_{0}\right] g_{0}\right),
\end{aligned}
$$




$$
\begin{aligned}
(g(\lambda), h) & =G_{3}(\lambda)+\left(\lambda-\lambda_{0}\right)\left(\mu_{0}-\lambda\right)^{-1}\left(E_{0}\left[\mu_{0}\right] g_{0}, h\right), \\
Q_{1}(\lambda) & =G_{4}(\lambda)+\left(\lambda-\lambda_{0}\right)\left(\lambda-\bar{\lambda}_{0}\right)\left(\mu_{0}-\lambda\right)^{-1}\left(E_{0}\left[\mu_{0}\right] g_{0}, g_{0}\right) .
\end{aligned}
$$

Here $G_{1}(\lambda), G_{2}(\lambda), G_{3}(\lambda)$ and $G_{4}(\lambda)$ are analytic in a neighborhood of $\mu_{0}$. By the symbol $E_{0}\left[\mu_{0}\right]$ we denote $E_{0}\left(\mu_{0}+0\right)-E_{0}\left(\mu_{0}-0\right)$.

Using equations (12), (13), (14) and (15), we see that

$$
\begin{aligned}
& Q_{1}^{2}(\lambda)+1=G_{5}(\lambda)\left(\mu_{0}-\lambda\right)^{-1}+\left(\lambda-\lambda_{0}\right)^{2}\left(\lambda-\bar{\lambda}_{0}\right)^{2}\left(\mu_{0}-\lambda\right)^{-2} \\
& \cdot\left(E_{0}\left[\mu_{0}\right] g_{0}, g_{0}\right)^{2} \text {, } \\
& \left(R_{0}(\lambda) f, h\right)\left[Q_{1}^{2}(\lambda)+1\right] \\
& =G_{6}(\lambda)\left(\mu_{0}-\lambda\right)^{-2}+\left(\lambda-\lambda_{0}\right)^{2}\left(\lambda-\bar{\lambda}_{0}\right)^{2} \\
& \cdot\left(\mu_{0}-\lambda\right)^{-3}\left(f, E_{0}\left[\mu_{0}\right] g_{0}\right)\left(E_{0}\left[\mu_{0}\right] g_{0}, h\right)\left(E_{0}\left[\mu_{0}\right] g_{0}, g_{0}\right) \text {, } \\
& (f, g(\bar{\lambda}))(g(\lambda), h)=G_{7}(\lambda)\left(\mu_{0}-\lambda\right)^{-1}+\left(\lambda-\lambda_{0}\right)\left(\lambda-\bar{\lambda}_{0}\right)\left(\mu_{0}-\lambda\right)^{-2} \\
& \cdot\left(f, E_{0}\left[\mu_{0}\right] g_{0}\right)\left(E_{0}\left[\mu_{0}\right] g_{0}, h\right) \text {, } \\
& Q_{1}(\lambda)(f, g(\bar{\lambda}))(g(\lambda), h) \\
& =G_{8}(\lambda)\left(\mu_{0}-\lambda\right)^{-2}+\left(\lambda-\lambda_{0}\right)^{2}\left(\lambda-\bar{\lambda}_{0}\right)^{2} \\
& \cdot\left(\mu_{0}-\lambda\right)^{-3}\left(f, E_{0}\left[\mu_{0}\right] g_{0}\right)\left(E_{0}\left[\mu_{0}\right] g_{0}, h\right)\left(E_{0}\left[\mu_{0}\right] g_{0}, g_{0}\right) \text {, }
\end{aligned}
$$

where $G_{5}(\lambda), G_{6}(\lambda), G_{7}(\lambda), G_{8}(\lambda)$ are analytic in a neighborhood of $\mu_{0}$.

Substituting (16), (17), (18), (19) into (6) and (7), we obtain

$$
\begin{aligned}
F_{1}(\lambda)= & {\left[G_{6}(\lambda)-G_{8}(\lambda)\right] } \\
\cdot & {\left[G_{5}(\lambda)\left(\mu_{0}-\lambda\right)+\left(\lambda-\lambda_{0}\right)^{2}\left(\lambda-\bar{\lambda}_{0}\right)^{2}\left(E_{0}\left[\mu_{0}\right] g_{0}, g_{0}\right)^{2}\right]^{-1}, } \\
F_{2}(\lambda)= & {\left[G_{7}(\lambda)\left(\mu_{0}-\lambda\right)+\left(\lambda-\lambda_{0}\right)\left(\lambda-\bar{\lambda}_{0}\right)\left(f, E_{0}\left[\mu_{0}\right] g_{0}\right)\left(E_{0}\left[\mu_{0}\right] g_{0}, h\right)\right] } \\
\cdot & {\left[G_{5}(\lambda)\left(\mu_{0}-\lambda\right)+\left(\lambda-\lambda_{0}\right)^{2}\left(\lambda-\bar{\lambda}_{0}\right)^{2}\left(E_{0}\left[\mu_{0}\right] g_{0}, g_{0}\right)\right]^{-1} . }
\end{aligned}
$$

From these expressions we see that $F_{1}(\lambda)$ and $F_{2}(\lambda)$ are analytic in a neighborhood of an eigenvalue $\mu_{0}$.

This establishes our claim that $F_{1}(\lambda)$ and $F_{2}(\lambda)$ are entire functions of $\lambda$.

Let $E(\mu)$ be the spectral function of $A$ corresponding to the selfadjoint extension or dilation $A^{+}$. By the Stieltjes inversion formula, for all $f, h$ in $H$ and for any real numbers $\alpha, \beta, \alpha<\beta$,

$$
\begin{aligned}
\left(\left[\frac{1}{2}\{E(\beta)+E(\beta+0)\}-\frac{1}{2}\{E(\alpha)+E(\alpha+0)\}\right] f, h\right) \\
\quad=(2 \pi i)^{-1} \lim _{\tau \rightarrow+0} \int_{\alpha}^{\beta}([R(\sigma+i \tau)-R(\sigma-i \tau)] f, h) d \sigma .
\end{aligned}
$$

Substituting (5) into (20), we obtain 


$$
\begin{aligned}
\left(\left[\frac{1}{2}\{E(\beta)+E(\beta+0)\}-\frac{1}{2}\{E(\alpha)+E(\alpha+0)\}\right] f, h\right) \\
=(2 \pi i)^{-1} \lim _{\tau \rightarrow+0} \int_{\alpha}^{\beta}\left[F_{1}(\sigma+i \tau)-F_{1}(\sigma-i \tau)\right] d \sigma \\
+(2 \pi i)^{-1} \lim _{\tau \rightarrow+0} \int_{\alpha}^{\beta}\left\{F_{2}(\sigma+i \tau)\left[\theta(\sigma+i \tau) Q_{1}(\sigma+i \tau)-1\right]\right. \\
\cdot\left[\theta(\sigma+i \tau)+Q_{1}(\sigma+i \tau)\right]^{-1} \\
-F_{2}(\sigma-i \tau)\left[\theta(\sigma-i \tau) Q_{1}(\sigma-i \tau)-1\right] \\
\left.\cdot\left[\theta(\sigma-i \tau)+Q_{1}(\sigma-i \tau)\right]^{-1}\right\} d \sigma .
\end{aligned}
$$

The first limit is zero by the analyticity of $F_{1}(\lambda)$. Since $\theta(\bar{\lambda})$ $=[\theta(\lambda)]^{-}, Q_{1}(\bar{\lambda})=\left[Q_{1}(\lambda)\right]^{-}$and $I Q_{1}(\lambda)>0$ for $I \lambda>0$ (see [3]), we may put $\Psi(\lambda)=F_{2}(\lambda)$ and $\Phi(\lambda)=\left[\theta(\lambda) Q_{1}(\lambda)-1\right]\left[\theta(\lambda)+Q_{1}(\lambda)\right]^{-1}$ in Lemma 1 in order to evaluate the second limit. Hence,

$$
\begin{gathered}
\left(\left[\frac{1}{2}\left\{E^{+}(\beta)+E^{+}(\beta+0)\right\}-\frac{1}{2}\left\{E^{+}(\alpha)+E^{+}(\alpha+0)\right\}\right] f, h\right) \\
=\left(\left[\frac{1}{2}\{E(\beta)+E(\beta+0)\}-\frac{1}{2}\{E(\alpha)+E(\alpha+0)\}\right] f, h\right) \\
=\int_{\alpha}^{\beta}(f, g(\sigma))(g(\sigma), h)\left[Q_{1}^{2}(\sigma)+1\right]^{-1} d \rho(\sigma),
\end{gathered}
$$

where $\rho(\sigma)=(1 / \pi) \lim _{\tau \rightarrow+0} \int_{0}^{\sigma} I \Phi(\mu+i \tau) d \mu$, and $E^{+}(\mu)$ is the spectral function of $A^{+}$.

It is understood that the integrand is defined by continuity at the eigenvalues of $A_{0}$. From (21) follows equation (4).

Since $A^{+}$is a minimal selfadjoint extension, the Hilbert space $H^{+}$ in which $A^{+}$acts is the closed linear hull of the set $Z$ of elements of the form $\left[E^{+}(\beta)-E^{+}(\alpha)\right] f$ for arbitrary $\alpha, \beta$ and arbitrary $f$ in $H$. (See NaImark [7].)

Let $\left\{\mu_{k}\right\}$ be the eigenvalues of $A_{0}$ arranged in order of growth. If $|k|$ is even, let $Q_{2}(\sigma)=\left[Q_{1}^{2}(\sigma)+1\right]^{1 / 2}$ for $\mu_{k}<\sigma<\mu_{k+1}$; if $|k|$ is odd, let $Q_{2}(\sigma)=-\left[Q_{1}^{2}(\sigma)+1\right]^{1 / 2}$ for $\mu_{k}<\sigma<\mu_{k+1}$. If the eigenvalues are bounded above or below, we modify the above definition accordingly for $\sigma$ greater than the largest eigenvalue or for $\sigma$ less than the smallest eigenvalue. The definition of $Q_{2}(\sigma)$ has been contrived so that $(f, g(\sigma))\left[Q_{2}(\sigma)\right]^{-1}$ is a continuous function of $\sigma$.

We define an operator $V$ on $Z$ into $L_{\rho}^{2}$ as follows: $V\left[E^{+}(\beta)-E^{+}(\alpha)\right] f$ $=\chi_{[\alpha, \beta)}(\sigma)(f, g(\sigma))\left[Q_{2}(\sigma)\right]^{-1}$, where $\chi_{[\alpha, \beta)}(\sigma)$ is the characteristic function of $[\alpha, \beta)$. From (4) we see that $\left\|V\left[E^{+}(\beta)-E^{+}(\alpha)\right] f\right\|$ $=\left\|\left[E^{+}(\beta)-E^{+}(\alpha)\right] f\right\|$. We now extend $V$ linearly to the linear hull of $Z$ and by continuity to all of $H^{+} . V$ is then an isometry of $H^{+}$into $L_{\rho}^{2}$. $V$ is in fact onto, because the set $V Z$ is dense in $L_{\rho}^{2}$. To see this, sup- 
pose that $k(\sigma) \in L_{\rho}^{2}$ and that $k(\sigma)$ is perpendicular to $\chi_{[\alpha, \beta)}(\sigma)(f, g(\sigma))$ - $\left[Q_{2}(\sigma)\right]^{-1}$ for all intervals $[\alpha, \beta)$ and for all $f$ in $H$. As in $[3]$ it follows that $k(\sigma)=0$ almost everywhere with respect to $\rho$ for points in the resolvent set of $A_{0}$. If $\mu_{0}$ is an eigenvalue of $A_{0}$, we can show that

$$
\int_{\left[\mu_{0}\right]}(f, g(\sigma))\left[Q_{2}(\sigma)\right]^{-1} k(\sigma) d \rho(\sigma)=0,
$$

i.e.,

$$
\left(f, g\left(\mu_{0}\right)\right)\left[Q_{2}\left(\mu_{0}\right)\right]^{-1} k\left(\mu_{0}\right) \Delta \rho\left(\mu_{0}\right)=0,
$$

where $\Delta \rho\left(\mu_{0}\right)$ is the $\rho$-measure of $\mu_{0}$. Taking $f=g_{0}$ in this last equation and using the fact that $\left(g_{0}, g\left(\mu_{0}\right)\right)\left[Q_{2}\left(\mu_{0}\right)\right]^{-1} \neq 0$, we see that $k\left(\mu_{0}\right)=0$ if $\Delta \rho\left(\mu_{0}\right) \neq 0$. (Note that by $\left(f, g\left(\mu_{0}\right)\right)\left[Q_{2}\left(\mu_{0}\right)\right]^{-1}$ we mean the value of the function $(f, g(\sigma))\left[Q_{2}(\sigma)\right]^{-1}$ defined by continuity at $\sigma=\mu_{0}$.) Hence, $k(\sigma)=0$ almost everywhere with respect to $\rho$. This shows that $V Z$ is dense in $L_{\rho}^{2}$.

It is not difficult to check that $V$ carries the spectral function of $A^{+}$into the spectral function of the multiplication operator in $L_{\rho}^{2}$. This proves the theorem.

REMARK 1. Suppose $A^{+}$is a selfadjoint extension of $A$ in $H$ (different from $A_{0}$ ), so that the function $\theta(\lambda)$ in equation (3) is identically equal to a real constant $\theta_{0}$. Then from equation (2) for $\rho(\sigma)$ and the fact that $A^{+}$is unitarily equivalent to the multiplication operator in $L_{\rho}^{2}$, we easily deduce the well-known results that the spectrum of $A^{+}$ consists of eigenvalues with no finite limit point and that the eigenvalues of $A^{+}$are all different from those of $A_{0}$. From this it follows that every point is of regular type for $A$.

REMARK 2. Let $F(\lambda)=\left[\theta(\lambda)-i I \lambda_{0}\right]\left[\theta(\lambda)+i I \lambda_{0}\right]^{-1}$ for $I \lambda>0$. Then $F(\lambda)$ is analytic in the upper half-plane and $|F(\lambda)| \leqq 1$. Suppose that $F(\lambda)$ is continuous down to the real axis and that $|F(\lambda)|<1$ on the real axis. Then one may check that $\Phi(\lambda)$ is continuous (i.e., may be defined continuously) down to the real axis, and $I \Phi(\lambda)$ is positive on the real axis. Hence, $\rho(\mu)=(1 / \pi) \int_{0}^{\sigma} I \Phi(\mu) d \mu$ is absolutely continuous, and the multiplication operator in $L_{\rho}^{2}$ is unitarily equivalent to the multiplication operator in $L^{2}$. Thus, if $F(\lambda)$ is continuous down to the real axis and $|F(\lambda)|<1$ on the real axis, it follows that $A^{+}$is unitarily equivalent to the multiplication operator in $L^{2}$.

REMARK 3. If $A$ is a singular Sturm-Liouville operator, one may use the work of $\mathrm{Kac}$ [4, Theorem 7] in order to consider the case that there exists a selfadjoint extension $A_{0}$ with a singular spectral function. Let $A$ be the symmetric operator in $L^{2}[0, \infty)$ generated by the differential operator $l[y]=-d^{2} y / d x^{2}+q(x) y$ where $q$ is real and con- 
tinuous for $0 \leqq x<\infty$. Suppose that the differential operator is in the limit point case and that the spectral function $\rho_{0}(\sigma)$ corresponding to the Weyl function $m(\lambda)$ is singular (i.e., $A$ has a selfadjoint extension $A_{0}$ with a singular spectral function). According to Straus [9] there is a one-one correspondence between the minimal selfadjoint dilations $A^{+}$of $A$ and the class of functions $\theta(\lambda)$ which are analytic in the upper half-plane and have nonnegative imaginary part. If $E(\lambda)$ is the spectral function of $A$ corresponding to the minimal selfadjoint extension $A^{+}$, then by the work of $\mathrm{Kac}$, for $f \in L^{2}(0, \infty)$,

$$
\begin{gathered}
{\left[\frac{1}{2}\{E(\beta)+E(\beta+0)\}-\frac{1}{2}\{E(\alpha)+E(\alpha+0)\}\right] f(x)} \\
=\int_{\alpha}^{\beta} \int_{0}^{\infty} f(y) u(y, \sigma) d y u(x, \sigma) d \rho(\sigma)
\end{gathered}
$$

where

$$
\begin{aligned}
& \rho(\sigma)=(1 / \pi) \lim _{\tau \rightarrow+0} \int_{0}^{\sigma} I \Phi(\mu+i \tau) d \mu, \\
& \Phi(\lambda)=[\theta(\lambda) m(\lambda)-1][\theta(\lambda)+m(\lambda)]^{-1},
\end{aligned}
$$

and $u(x, \sigma)$ is a solution of $l[y]-\sigma y=0, y(0)+m(\sigma) y^{\prime}(0)=0$. The latter equation is to be interpreted as $y^{\prime}(0)=0$ in the event that $\lim _{\lambda \rightarrow 0} m(\lambda)$ does not exist as a finite number.

\section{REFERENCES}

1. N. I. Achieser and I. M. Glasmann, Theorie der linearen operatoren im HilbertRaum, Akademie-Verlag, Berlin, 1954.

2. E. A. Coddington and R. C. Gilbert, Generalized resolvents of ordinary differential operators, Trans. Amer. Math. Soc. 93 (1959), 216-241.

3. R. C. Gilbert, Spectral multiplicity of selfadjoint dilations, Proc. Amer. Math. Soc. 19 (1968), 477-482.

4. I. S. Kac, Spectral multiplicity of a second-order differential operator and expansion in eigenfunctions, Izv. Akad. Nauk SSSR Ser. Mat. 27 (1963), 1081-1112.

5. M. G. KreYn, The theory of self-adjoint extensions of semibounded hermitian transformations and its applications. I, Mat. Sb. 20 (62) (1947), 431-495.

6. R. McKelvey, The spectra of minimal selfadjoint extension of a symmetric operator, Pacific J. Math. 12 (1962), 1003-1022.

7. M. A. NaImark, Spectral functions of a symmetric operator, Izv. Akad. Nauk SSSR Ser. Mat. 4 (1940), 277-318.

8. A. V. Straus, On spectral functions of differential operators, Izv. Akad. Nauk SSSR Ser. Mat. 19 (1955), 201-220.

9. - Expansion by eigenfunctions of a second-order boundary problem on a half-line, Izv. Akad. Nauk SSSR Ser. Mat. 20 (1956), 783-792.

California State College, Fullerton 